\section{Bridging the Gap Between Pharmacoeconomics and the Real-World Practice of Managed Care Pharmacy}

Drug coverage decisions made by managed care payers have the potential to influence millions of lives at a time. The importance of making "evidence-based" coverage decisions has never been greater, particularly since the implementation of Medicare Part D in January 2006. For the first time since the inception of Medicare in 1965, all Medicare beneficiaries have access to drug coverage, albeit in different coverage schemes with some variation in out-ofpocket expense.

The influx of about 50 million managed care members from Medicare Part D into the overall managed care pharmacy market further amplifies the significance of any coverage decision. Currently about 250 million Americans obtain their medications through managed care plans. ${ }^{1}$ While medication therapy management has emerged as a framework to streamline the determination of optimal treatment options at the individual level, there remains a need for a universally accepted framework for the clinical and economic evaluation of therapies at the population level for drug coverage policies. In addition to examining the safety and cost-effectiveness of drugs, plans need to consider the implications of copayments, deductibles, and prior authorization requirements for access to new drugs.

Within an increasingly competitive landscape, there is a delicate balance between the access and cost implications of coverage decisions because patients, as imperfectly informed consumers, will be concerned about what they pay for coverage and what benefits they receive as a result. At no other time have the questions of drug benefit design and formulary decisions been so critical for managed care plans and their enrollees. Indeed, this new Medicare benefit has arguably made the relationship between premiums and out-of-pocket costs for prescription drugs more visible to millions of Americans.

Optimal coverage decisions require evidence that is timely, relevant, and adaptable to the specific population characteristics of the health plan. Perhaps most importantly, the evidence should be transparent to all stakeholders, particularly the members of the pharmacy and therapeutics (P\&T) committee. The goal of transparency is sometimes difficult to achieve, especially with respect to the economic evaluation of innovative therapies for complex, multifaceted chronic conditions such as diabetes. Such economic evaluations often rely on pharmacoeconomic models that project the likely costs and outcomes of new therapies over time, based on safety and efficacy data from phase 3 trials and data from numerous secondary sources, through a complex set of mathematical relationships among model variables. These complex models employ many interrelated assumptions, making it difficult to identify the precise process through which any particular assumption affects model projections.

In the current issue of JMCP, Spooner et al. found that 25\% of manufacturer dossiers received by a large health plan did not contain a pharmacoeconomic model for a new product, and
$45 \%$ of class review dossiers did not contain a pharmacoeconomic model. ${ }^{2}$ Receipt of a dossier, with or without a pharmacoeconomic model, did not appear to influence the outcome of the drug formulary decision by the P\&T committee for the health plan. ${ }^{3}$ In contrast, in the previous issue of JMCP, Watkins and colleagues provide a case study of a complex Markov modelthe CORE diabetes model - that was employed to inform formulary decisions relating to the prevention and control of diabetes in a managed care plan. This case study from Watkins et al. provides a good example of how information can be organized, analyzed, and presented to guide decision making. However, the question of how to raise the level of fluency with such models in managed care still remains to be answered.

This example also serves to underscore the numerous caveats that result from model assumptions required in such analyses. The authors acknowledge, for example, the design weakness of using a 30-year projection, given the irrelevance of this time frame to managed care, but more importantly, the lack of safety data on such cumulative exposure, if it were plausible. They also admit that they may have overestimated the benefit of weight loss, based on the first-year estimate alone. Attrition bias inherent in the numbers from the intent-to-treat analyses appears to be another source of possible error. ${ }^{4}$

An increasing share of health budgets is being consumed by chronic disease, and this is only expected to increase, given the demographics of Medicare beneficiaries and the epidemiology of these diseases. For instance, more than 10 million Americans are diagnosed with type 2 diabetes, with an additional 5.5 million estimated to have the disease but who have not yet been diagnosed. ${ }^{5}$ Diabetes has been identified as a significant risk factor for the development of heart failure, a disease with major public health implications ${ }^{6.7}$ Clearly, the potential impact of an efficient clinical and economic evaluation framework can have major budget implications for managed care plans.

As Watkins et al. note, "Many health plans now use the AMCP Format as a tool to improve efficiency in gathering clinical information, but relatively few decision makers give serious consideration to the [pharmacoeconomic] models offered with product dossiers," often due to the fact that the majority of plans still do not know what to do with this information. In some cases, the problem is that pharmacoeconomic models are not considered credible because of the use of implausible or unsubstantiated model assumptions. In other cases, the model results are not considered informative because of the selection of an irrelevant comparator for the new treatment. Lack of transparency also contributes to the lack of credibility_many pharmacoeconomic models resemble a "black box" where model assumptions are fed into one end, "magic" happens, and results emerge from the other end. In the absence of transparency, health plans are likely to suspect that the results produced by models reflect some form of hidden bias, which impairs credibility. 
We have seen the need for "translational research" in clinical medicine, and perhaps this is what is needed now in managed care pharmacy. We need to bridge the gap between pharmacoeconomics and actual pharmacy management practice. The "number needed to treat" measure for example can be intuitive to managed care due to its focus on population care management. Similarly, pharmacoeconomic models using "cost per diseaseevent avoided" as a cost-effectiveness metric may be more intuitive for managed care when making coverage decisions among alternative treatments for a particular condition than the metric of "costs per quality-adjusted life-year gained" preferred by methodological purists. ${ }^{8,9}$

An alternative to using economic evaluations in product dossiers based on industry-sponsored pharmacoeconomic models is to use economic evaluations produced by third parties, such as the United Kingdom's National Institute for Health and Clinical Excellence (NICE). This approach may alleviate some of the inherent limitations-such as in the example presented by Watkins et al._of a model application developed by industry, presumably for a commercial purpose. However, such thirdparty evaluations for new products may not be available within the necessary time frame for a particular health plan's formulary decision. Further, results of third-party evaluations often must be adapted for the specific populations or clinical issues relevant for a formulary decision within a particular managed care plan.

In the absence of timely economic evaluation from independent third parties, managed care plans could, of course, develop their own pharmacoeconomic models, but in many cases, this option will be prohibitively expensive, time consuming, and require expertise not readily available to the health plan. Thus, communication between managed care plans and pharmaceutical companies to generate pharmacoeconomic information regarded as credible and relevant can, under the right circumstances, enable health plans to make more informed and timely formulary decisions.

In the example presented by Watkins et al., a proactive effort by a pharmaceutical company to understand a payer's modeling needs resulted in the development of pharmacoeconomic information that the payer regarded as credible and meaningful for its population. However, a practical limitation of this example is that different managed care organizations will have different concepts of what specific model characteristics contribute to credible or useful pharmacoeconomic information. Therefore, given the diversity of managed care organizations, pharmacoeconomic models provided by pharmaceutical companies must be flexible, particularly in the definition of relevant population characteristics and in selection of the specific treatment comparators.

Unfortunately, the modeling framework used in the example presented by Watkins et al. is relatively inflexible. In addition, many fixed data inputs are speculative and perhaps erroneous, and some assumptions employed by the researchers impractical. The impact of these potentially erroneous assumptions on model results is not adequately illustrated or made explicit using sensitivity analyses. The description of the model is not sufficiently transparent to enable a reader to assess the implications of these assumptions. Further, although this modeling effort was regarded as useful by the health plan in this example, the ultimate formulary decision was to impose almost none of the criteria for use of the drug suggested by the pharmacoeconomic model. Finally, the lack of transparency and flexibility in the modeling approach in this example limits the potential for its immediate use for formulary decisions by other health plans.

Fadia T. Shaya, PhD, MPH Associate Professor, Associate Director Center on Drugs and Public Policy University of Maryland School of Pharmacy Pharmaceutical Health Services Research 220 Arch St., 12th Fl. Baltimore, MD 21201 fshaya@rx.umaryland.edu

Robert L. Ohsfeldt, PhD Professor, Health Policy \& Management School of Rural Public Health Texas A\&M Health Science Center College Station, TX rohsfeldt@srph.tamhsc.edu

\section{DISCLOSURES}

The authors disclose no potential bias or conflict of interest relating to this article.

\section{REFERENCES}

1. Available at: http://www.amcp.org/amcp.ark. Accessed January 9, 2007.

2. Spooner JJ, Gandhi PK, Connelly SB. AMCP Format dossier requests: manufacturer response and formulary implications for one large health plan. J Manag Care Pharm. 2006;13(1):37-43.

3. Watkins JB, Minshall ME, Sullivan SD. Application of economic analyses in U.S. managed care formulary decisions: a private payer's experience. J Manag Care Pharm. 2006;12(9):726-35. Available at:http://www.amcp.org/data/jmcp/ 726-735.pdf. Accessed December 19, 2006.

4. Blonde L, Klein EJ, Han J. Interim analysis of the effects of exenatide treatment on Alc, weight and cardiovascular risk factors over 82 weeks in 314 overweight patients with type 2 diabetes. Diabetes Obes Metab. 2006;8:436-47.

5. Office of Disease Prevention and Health Promotion, Office of Public Health and Science, U.S. Department of Health \& Human Services: Healthy People 2010: Objectives for Improving Health. P. 5.3-5.39. Available at: http://www. healthypeople.gov/Document/pdf/uih/2010uih.pdf. Accessed December 19, 2006.

6. He J, Ogden LG, Bazzano L, et. al. Risk factors for congestive heart failure in US men and women: NHANES I epidemiologic follow-up study. Arch Intern Med. 2001;161(7):996-1002.

7. Aronow WS, Ahn C. Incidence of heart failure in 2,737 older persons with and without diabetes mellitus. Chest. 1999;155(3):867-68.

8. Gold MR, Siegel JE, Russell LB, Weinstein MC, eds. Cost-effectiveness in Health and Medicine. Oxford, U.K.: Oxford University Press; 1996.

9. McGregor M. Cost-utility analysis: Use QALYs only with great caution. CMAJ. 2003;168(4):433-34. 